\title{
Fraudès detectadas na comercialização de pescado no município de Niterói, Estado do Rio de Janeiro, Brasil
}

\author{
Frauds detected in fresh fish commercialized in Niterói, State of \\ Rio de Janeiro, Brazil
}

Francisco Carlos de Lima, Eliana de Fátima Marques de Mesquita

\section{Resumo}

A presente pesquisa procurou identificar as fraudes mais comuns no comércio varejista de pescado do Estado do Rio de Janeiro. Foi baseada na observação das espécies comercializadas em bancas varejistas, que foram fotografadas após a constatação da fraude e identificadas cientificamente. Das doze fraudes observadas, nove eram por mudança de denominação dos peixes inteiros ou de suas partes. Apenas uma fraude grosseira, quanto ao peso, foi detectada em lulas, e outra em filés de sardinhas oferecidos já em início de deterioração.

Palavras chave: fraude; pescado; comercialização

\section{Introdução}

A rigor, fraude é tudo o que se desvia das características normais, embora este termo seja um tanto vago e discutível do ponto de vista comercial e industrial. Assim, podem ser considerados fraude os artifícios usados sem o consentimento oficial e que não fazem parte de uma prática universalmente aceita (Riedel, 1992).

As fraudes em alimentação, além de importarem em infrações flagrantes à ética indústrio-comercial em desrespeito aos padrões oficialmente adotados, promovem, nos alimentos, modificações organolépticas, de composição química, do equilíbrio percentual de seus constituintes normais e, ainda, a quebra das boas condições do seu estado sanitário, acarretando, aos consumidores, prejuízos não só de ordem biológica, como também de ordèn econômica (Earbosa.; 1976).

A Constituição Federal de 1988, em seu Art. 50, inciso XXXII, p. 8, diz: "o Estado promoverá, na forma da lei, a defesa do consumidor" (Brasil, 1988). Em decorrência deste e de outros preceitos constitucionais, o Congresso Nacional aprovou a Lei Federal no 8.078 de 11 de setemfro le 1990, o dito Código de Defesa do Consumidor, que está em vigor desde março de 1991 (Brasil, 1990).

Este trabalho teve por objetivo a identificação das fraudes mais comuns observadas nos mercados varejistas de pescado do município de Niterói, Estado do Rio de Janeiro.

\section{Material e Métodos}

A pesquisa foi constituída pela observação dos espécimes comercializados em bancas varejistas, entrepostos de pescado e peixarias.

Os espécimes foram fotografados, após a constatação da fraude, identificados segundo Figueiredo e Menezes $(1978,1980)$, Menezes e Figueiredo (1980), Menni et al. (1984) e Carvalho Filho (1992), e através de comunicação escrita aos autores, em outubro de 1995, pelos Professores Francisco J. de Figueiredo e Ulisses L. Gomes, do Departamento de Biologia Animal e Vegetal, Instituto de Biologia, Universidade do Estado do Rio de Janeiro UERJ.

\section{Resultados}

As fraudes mais comuns no mercado varejista referem-se às espécies de pescado agrupadas a seguir:

GRUPO I: MARIA-MOLE, GOETE, TIRA-VIRA, PESCADINHA

Devido à ampla sinonímia utilizada nas inúmeras regiões do país, há uma grande dificuldade para se definir as espécies de peixes, principalmente aquelas conhecidas popularmente como pescadinina. Porém, no município de Niterói e em outras áreas do Estado do Rio de Janeiro, os nomes vulgares relacionados neste grupo são de uso estabelecido no comércio de pescado.

Por ser a pescadinha um peixe mais conhecido e de maior aceitação pelo consumidor, sendo recomendada pelcs médicos para pessoas convalescentes, crianças e idosos, as demais espécies citadas são utilizadas pêlos comerciantes para fraudar o comprador. As características das espécies são:

MARIA-MOLE - Sciaenidae: Cynoscion striatus (Cuvier, 1829) - Espécie muito comum no litoral sudeste onde é capturada em grande quantidade na pesca comercial. Sua carne torna-se amolecida com grande facilidade, daí seu nome vulgar (Fig. 1a).

GOETE - Sciaenidae: Cynoscion jamaicensis (Vaillant \& Bocourt, 1883) - Espécie tipicamente marinha. Repre- 
senta um razoável recurso pesqueiro no litoral sudeste.

Tanto a Maria-mole quanto o Goete são fraudados inteiros, já que se assemelham à pescadinha.

TIRA-VIRA - Percophidae: Percophis brasiliensis Quoy \& Gaimard, 1824 - Espécie considerada como de excelente gosto, também conhecida como peixe aipim; relativamente comum no comércio, apresenta grande quantidade de espinhas acessórias na musculatura dorso-lateral, o que a torna inadequada para crianças e idosos. É fraudada filetada (Fig. 1b).

PESCADINHA - Sciaenidade: Isopisthus parvipinnis (Cuvier, 1830) - Embora pequena, é muito apreciada; tem excelente carne e grande valor comercial. É diferenciada das duas primeiras espécies por possuir escamas extremamente pequenas (Fig. 1c).
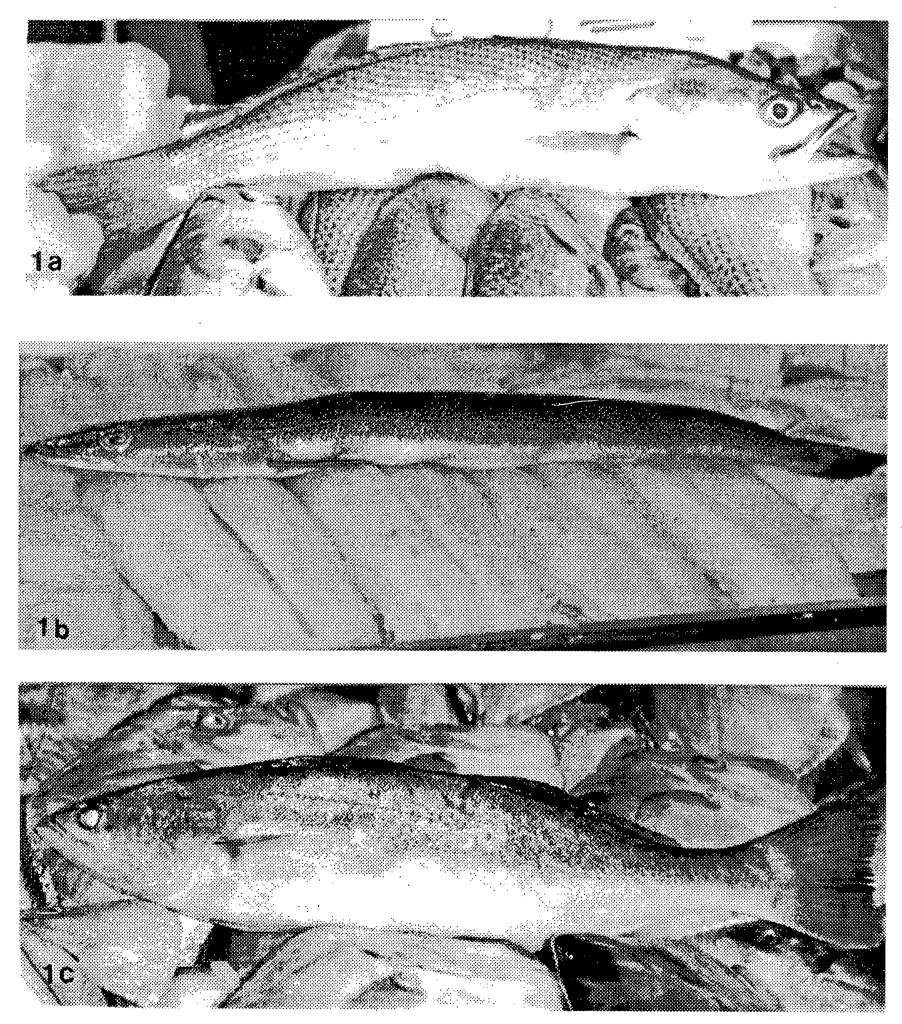

Figura la, ic - Maria Mole e Pescadinha, respectivamente. Notar a diferença de tamanho entre as escamas dos exemplares; $1 \mathrm{~b}$ - Tira-vira apoiado sobre filés obtidos de peixes da mesma espécie.

\section{GRUPO II: BATATANAMORADO BADFJO}

BATATA - Malacanthidae: Lopholatilus villarii Ribeiro, 1915 - Carne de bom paladar. Esta espécie apresenta pintas e manchas amarelas em todo o corpo (Fig. 2a); mesmo quando há tentativa de retirada, elas permanecem no dorso, na cauda e na cabeça, revelando a fraude (Figs. 2c, d).

NAMORADO - Pinguipedidae: Pseuclopercis semifasciata (Cuvier, 1829) - Apreciadíssimo, de alto va- lor, é comum no comércio. Tem coloração escura, com máculas brancas dorso-lateralmente e prateadas no ventre (Fig. 2b); sua carne é extremamente branca, o que a diferencia da carne do batata que tende para o róseo. Apresenta espinhas acessórias na musculatura.

BADEJO - Serranidae: Mycteroperca rubra (Bloch, 1793) - Excelente carne; é um dos peixes mais conhecidos e apreciados pelo consumidor.

Tanto o badejo quanto o namorado são vendidos quase que exclusivamente inteiros. O batata é usado na fraude de duas maneiras: os espécimes maiores são raspados com faca, eviscerados, descabeçados e posteados (Fig. 2c), sendo comercializados, na maioria das vezes, como badejo; os menores são eviscerados e posteados com cabeça (Fig. 2d), sendo vendidos como namorado. Além disso, a divisão em postas, efetuada em peixes já alterados, dificulta ou impede a observação das características organolépticas do peixe pelo consumidor.

\section{GRUPO III: ABRÓTEA/PESCADA}

ABRÓTEA - Gadidae: Urophycis brasiliensis (Kaup, 1858 ) - Comercialmente importante, congelado ou fres$c o$, é de muito bom sabor. Embora tenha carne de qualidade, não tem grande significado por ser espécie pouco conhecida no Rio de Janeiro (Fig. 3).

PESCADA - Sciaenidae: Macrodon ancylodon (Bloch \& Schneider, 1801) - Sabor semelhante ao da pescadinha, porém de maior valor comercial por seu tamanho. É bastante comum no litoral sul, aparecendo com freqüência nos mercados dessa região.

Nessa fraude, a abrótea é apresentada em filés, já que são espécies muito diferentes entre si; comercialmente é inferior à pescada.

\section{GRUPO IV: PEIXE-PESCADOR / LINGUADO}

PEIXE-PESCADOR - Lophiidae: Lophius gastrophysus Ribeiro, 1915 - É bentônico, alimenta-se de peixes menores. A espécie européia do gênero é muito apreciada como alimento em alguns países como Portugal, Espanha e Itália. A nossa espécie, pelo feio aspecto (Fig. 4b), não e táo apreciada; torna-se necessário o descabeçamento e a evisceração, a bordo dos barcos pesqueiros, visando uma melhor apresentação comercial. O Peixe-pescador, também conhecido como Peixe-diabo e Peixe-sapo, é exportado em grande quantidade rara eṇueles países eurof eus.

LINGUADO - Bothidae: Paralichthys brasilierisis (Ranzani, 1840) - Muitíssimo apreciado pelo sabor, é de alto valor comercial.

Esse tipo de fraude talvez seja o de mais fácil diagnóstico. O Peixe-pescador, uma espécie que envolve, após a sua captura, o descabeçamento e a evisceração, è apresentado nas bancas de comércio, sempre em filés enquanto que o Linguado é sempre vendido inteiro (Fig. 4a). 

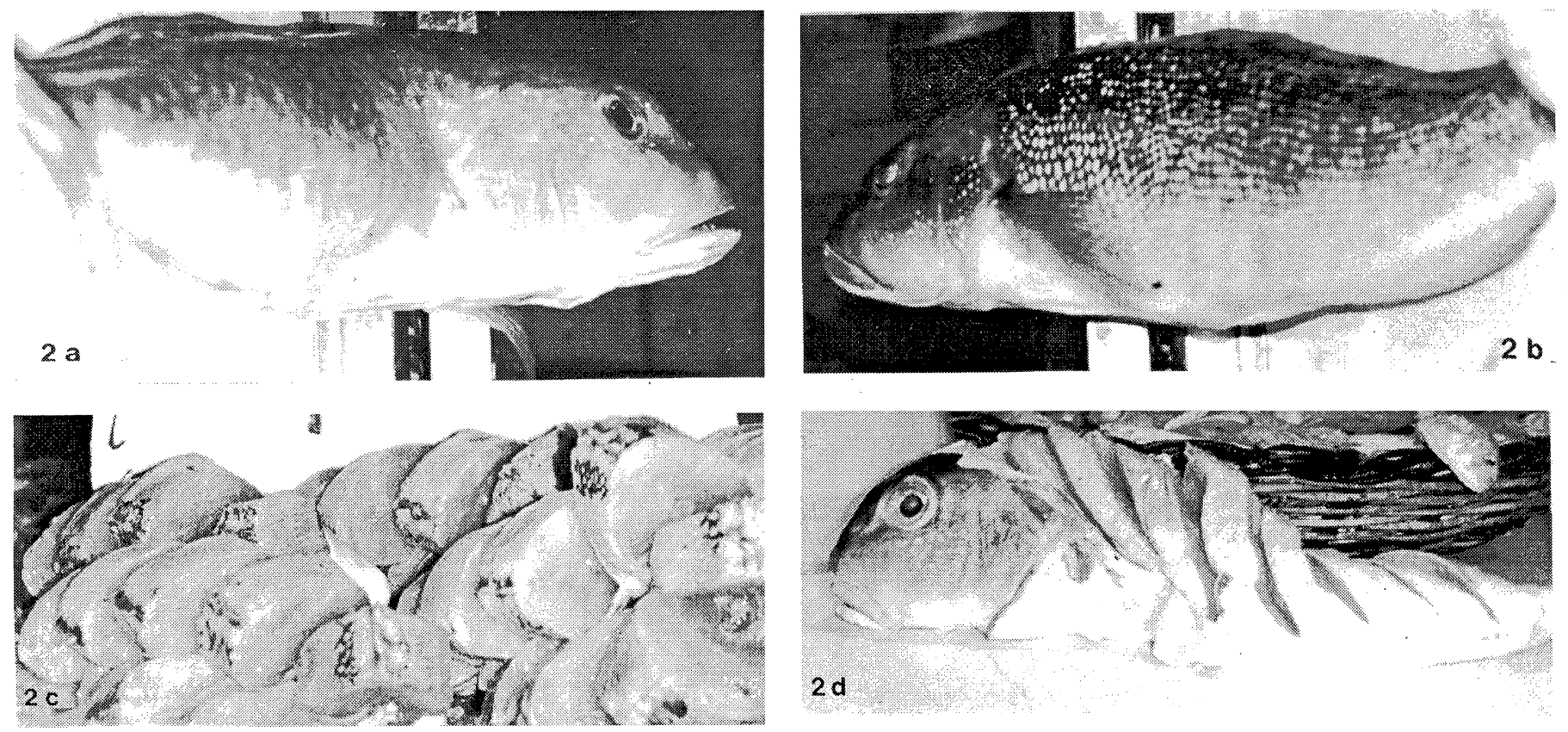

Figura 2a - Batata inteiro; 2c, 2d - Batata eviscerado e posteado sem cabeça e eviscerado e posteado com cabeça. As pintas e manchas podem ser facilmente observadas; $2 b$ - Namorado, com sua coloração característica (dorso escuro e ventre esbranquiçado).

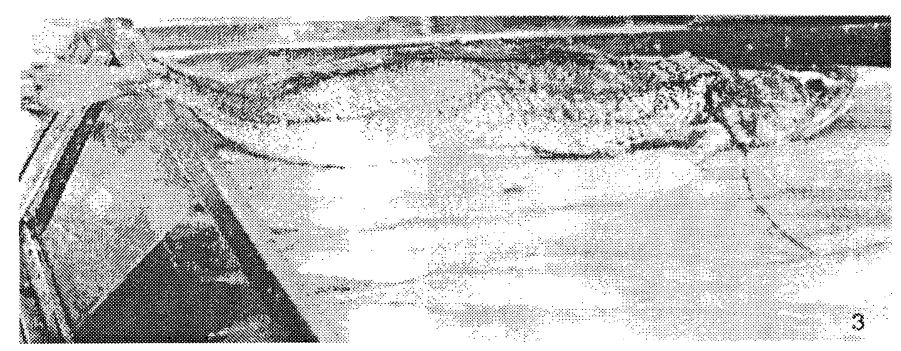

Figura 3 - Aspecto da Abrótea e dos seus filés.

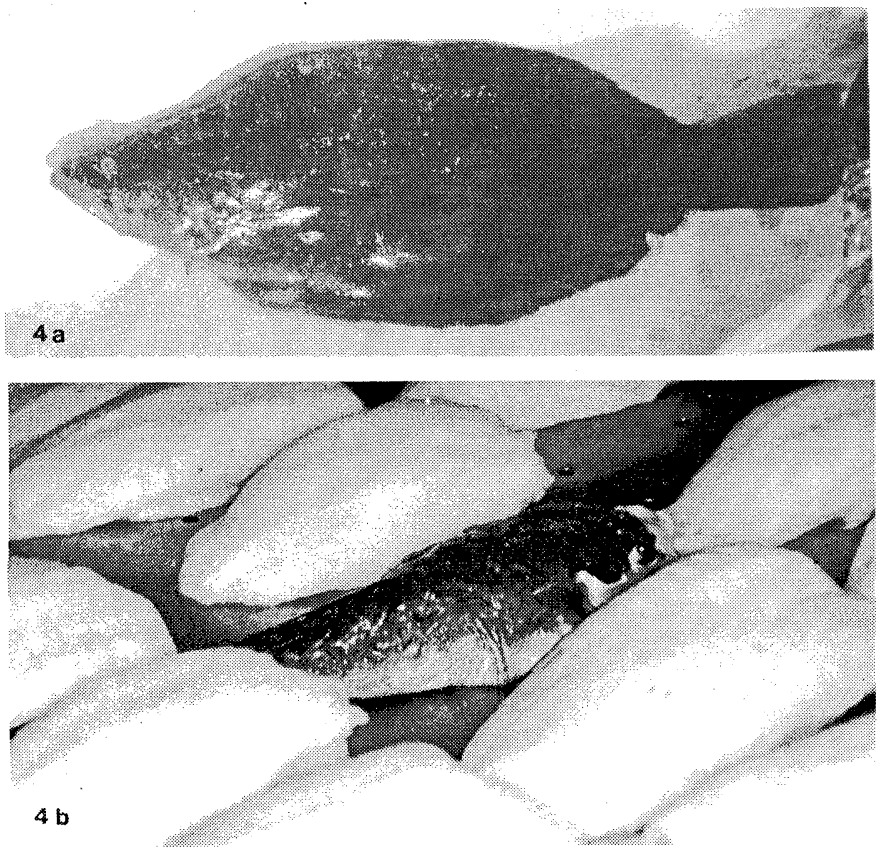

Figura 4a - Linguado; 4b - Peixe-sapo e os filés correspondentes.
GRUPO V: SARDINHA LAJE/SARDINHA VERDADEIRA

SARDINHA LAJE OU SARDINHA BANDEIRA Clupeidae: Opisthonema oglinum (Leseur, 1818) - Sabor pouco apreciado, chegando por vezes a substituir satisfatoriamente a sardinha; consumido basicamente por segmentos mais humildes da população.

SARDINHA VERDADEIRA - Clupeidae: Sardinella brasiliensis (Steindachner, 1879) (Fig. 5c) - Muito importante, sustenta grandes indústrias de enlatados; parte da produção vai direto ao consumidor, fresca, alimentando as classes menos favorecidas. No Sudeste do Brasil perfaz $30 \%$ do total do pescado embarcado. A pesca indiscriminada de peixes imaturos quase exterminou a espécie no Rio de Janeiro e São Paulo; por sua capacidade de reprodução, o estoque está se restabelecendo.

Essa fraude é realizada com o descabeçamento e um corte oblíquo na região ventral da Sardinha Laje, com o intuito de torná-la semelhante à verdadeira. A diferenciação se faz pela observação de um prolongamento do último raio da nadadeira dorsal, e de pintas escuras na linha da Sardinha Laje (Fig. 5a, b).

A Fig. $5 d$ mostra a forma de comercialização de sardinhas de médio e pequeno porte, quando no início da deterioração. Devido ao amolecimento da musculatura, torna-se fácil a retirada da coluna vertebral após sua evisceração.

\section{GRUPO VI: PARGO/VERMELHO}

PARGO - Sparidae: Pagrus pagrus (Linnaeus, 1758) Carne excelente, de grande valor comercial. Relativamente comum no litoral brasileiro (Fig. 6a). 

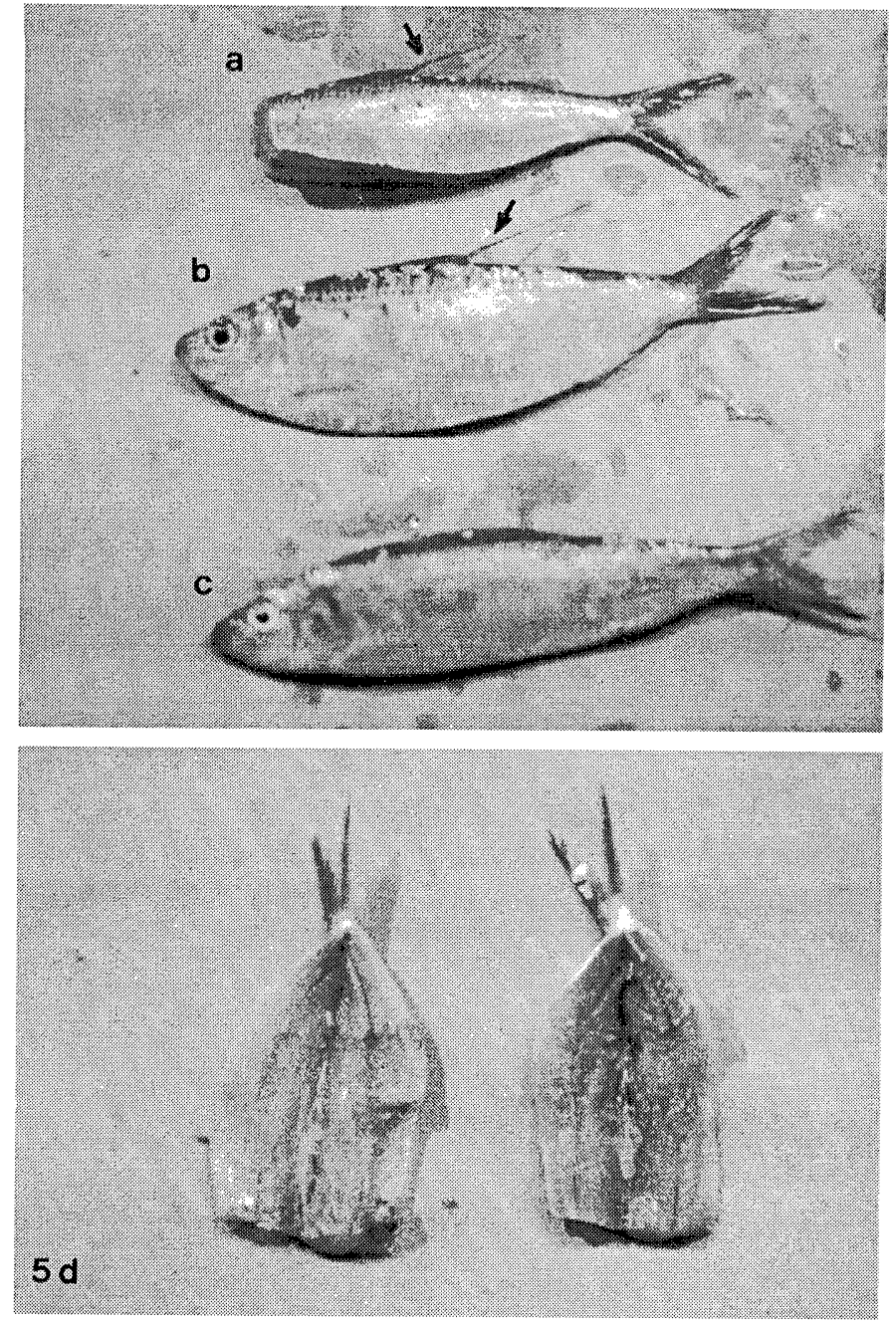

Figura 5a, b - Sardinha-laje cortada e inteira. As setas mostram o prolongamento do último raio da nadadeira dorsal. Notar também, imediatamente abaixo da linha lateral, as pintas escuras características; $5 \mathrm{c}$ Sardinha verdadeira: observa-se a sua forma mais alongada em relação à Sardinha-laje que é achatada e possui ainda ventre com borda cortante; 5d - Sardinha verdadeira eviscerada, descabeçada e palmeada.
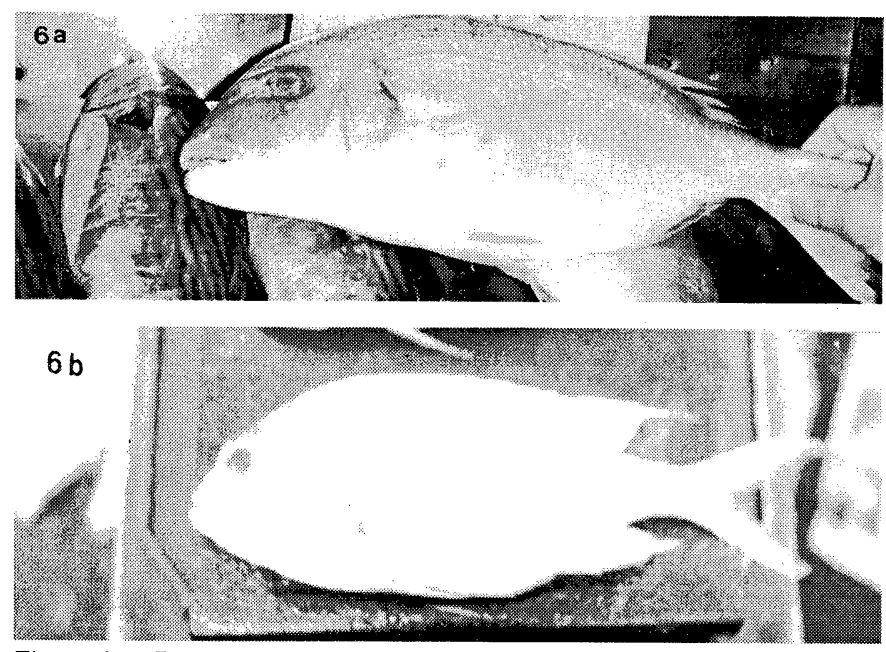

Figura 6a - Pargo; 6b - Vermelho.
VERMELHO - Lutjanidae: Lutjanus purpureus (Poey, 1875) - Tem grande importância comercial e sua carne é muito saborosa.

Esta fraude é facilmente detectada, pois o pargo é uma espécie de coloração levemente rosada e o vermelho, como o próprio nome indica, tem cor avermelhada (Fig. 6b).

\section{GRUPO VII: LULA RECHEADA}

LULA: Loliginidae: Dorytheutis brasiliensis (Blainville, 1823) - De grande importância comercial.

Denominada de fraude grosseira quanto ao peso, pois peixes pequenos são colocados dentro do manto da lula; este estratagema visa sonegar o peso e o volume do produto. É um tipo de fraude primária, fácil de ser detectada por um observador mais atento (Fig. 7).

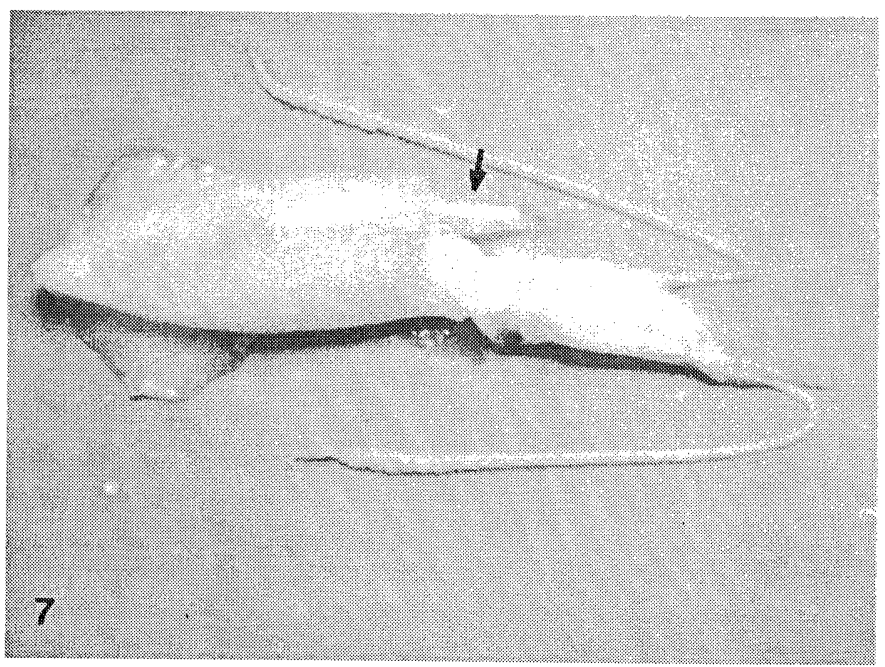

Figura 7 - Lula, com pequeno peixe introduzido no manto (seta).

\section{Discussão e Conclusões}

Pelo levantamento efetuado, observou-se que os trabalhos publicados nessa área são raros, o que dificulta a abordagem discussiva.

Muitas dessas fraudes são conhecidas, pelo menos dos órgãos de fiscalização sanitária, e podem ser classificadas como fraudes por falsificação e fraudes grosseiras quanto ao peso (segundo Barbosa, 1976; Evangelista, 1992; Riedel, 1992). As citadas e ilutradas no presente trabalho, foram:

a) mudança da denominação de certos peixes ou de suas partes, a fim de enquadrá-los em uma categoria mais cara, iludindo, assim, a economia popular;

b) a divisão, em postas, de peixes já alterados, com o intuito de impedir a observação das caracteristicas normais do peixe pelo comprador; e

c) introdução de elementos outros, estranhos ao produto, com o intuito de aumentar o seu peso.

As fraudes ocorrem devido à ignorância tanto do consumidor quanto dos responsáveis pela fiscalização. 


\section{Abstract}

Frauds detected in fresh fish commercialized in Niterói, State of Rio de Janeiro, Brazil

The study tried to identify the most ordinary frauds found in the fish market of Niterói, State of Rio de Janeiro.The commercialized species were observed and analyzed. Some photographs were taken from the species after scientific identificaton of each fraud. From twelve cases detected, nine were due to uncorrect denomination of the whole fishes or of parts of them. Only one coarse fraud involving weight was detected in squids. Sardines' flaps were commercialized in the very beginning of a deterioration process.

Key words: frauds; fishes; commercialization

\section{Referências bibliográficas}

BRASIL. Constituição: República Federativa do Brasil, 1988. Brasília: Senado Federal, 1988, 292p.

BRASIL. Lei Federal no 8.078, de 11 de setembro de 1990. Dispõe sobre a proteção do consumidor e dá outras providên- cias. Col. Leis Rep. Fed. Brasil. Brasília, v. 182, n. 5, p. 2829 2907, set./out. 1990.

BARBOSA, J.J. Introdução à Tecnologia de Alimentos. Rio de Janeiro: Kosmos, 1976, 118p.

CARVALHO FILHO, A. Peixes: costa brasileira. São Paulo: Marca D’Água, 1992, 304p.

EVANGELISTA, J. Tecnologia de Alimentos. São Paulo: Atheneu. 1992. 652p.

FIGUEIREDO, J.L., MENEZES, N.A. Manual de peixes marinhos do sudeste do Brasil. II. Teleostei (1). São Paulo: Museu de Zoologia, Universidade de São Paulo, 1978, 110p.

FIGUEIREDO, J.L., MENEZES, N.A. Manual de pexies marinhos do sudeste do Brasil. III. Teleostei (2). São Paulo: Museu de Zoologia, Universidade de São Paulo, 1980, 90p.

MENEZES, N.A., FIGUEIREDO, J.L. Manual de peixes marinhos do sudeste do Brasil. IV. Teleostei (3). São Paulo: Museu de Zoologia, Universidade de São Paulo, 1980, 96p.

MENNI, R.C., RINGUELET, R.A., ARAMBURU, R.H. Peces marinos de la Argentina y Uruguay. Catálogo crítico ilustrado. Clave para la determinación de familias, géneros y especies. Nombres vulgares. Glosario. Argentina: Editorial Hemisferio Sur, 1992, 320p.

RIEDEL, G. Controle sanitário dos alimentos. São Paulo: Atheneu, 1992, 320p. 